\title{
Verbal Support
}

National Cancer Institute

\section{Source}

National Cancer Institute. Verbal Support. NCI Thesaurus. Code C147981.

Therapy in which a therapist, or other personnel, provides nondirective, supportive care by offering supportive, validating statements and reflective listening. Verbal support sessions are aimed at providing an empathic, therapeutic environment to facilitate emotional expression and sharing of worries and fears. 